\title{
Incidência, Preditores e Impacto Clínico dos Sangramentos Maiores Associados à Intervenção Coronária Percutânea
}

\author{
Clarissa Campo Dall'Orto' ${ }^{1}$ Luiz Felipe Willi', Maria Sílvia Faraco Nogueira' ${ }^{1}$, Guilherme Alves Lapa', \\ João Batista de Oliveira Neto', Maria Fernanda Zuliani Mauro', Salvador André Bavaresco Cristóvão', \\ Adnan Ali Salman'1 , José Armando Mangione ${ }^{1}$
}

\section{RESUMO}

Introdução: O sangramento associado ao periprocedimento é uma das complicações mais freqüentes da intervenção coronária percutânea (ICP). O objetivo deste estudo foi avaliar a incidência, os preditores de sangramento maior e o impacto deste nos eventos adversos após ICP. Método: Realizada análise retrospectiva de 8.739 pacientes consecutivos submetidos a ICP eletiva no período de junho de 1997 a fevereiro de 2008. Comparamos os pacientes que apresentaram ou não sangramento maior (grupos SM e NSM, respectivamente) em relação às características clínicas e angiográficas e do procedimento, e a evolução intra-hospitalar e tardia. Resultados: Sangramento maior ocorreu em $1,6 \%$ dos pacientes. O modelo de regressão logística múltipla identificou como fatores preditores independentes de sangramento maior associado à ICP: sexo feminino, idade, cirurgia de revascularização miocárdica prévia e uso dos inibidores da glicoproteína $\mathrm{Ilb} / \mathrm{Illa}$. O sangramento maior periprocedimento foi preditor independente de insuficiência renal aguda, infarto agudo do miocárdio (IAM) e óbito na fase hospitalar e de IAM na fase tardia. Conclusão: Este estudo demonstra que o sangramento maior associado periprocedimento é preditor independente de eventos adversos nas fases precoce e tardia pós-ICP.

DESCRITORES: Hemorragia. Angioplastia transluminal percutânea coronária. Mortalidade.

\section{SUMMARY}

\author{
Incidence, Predictors and Clinical Impact \\ of Major Bleeding After Percutaneous \\ Coronary Intervention
}

Background: Bleeding is one of the most frequent complications of percutaneous coronary intervention $(\mathrm{PCI})$. The objective of this study was to evaluate the incidence and predictors of major bleeding and the impact of this complication on adverse events after $\mathrm{PCl}$. Method: A retrospective analysis of 8,739 consecutive patients who were submitted to elective $\mathrm{PCI}$ from June/1997 to February/ 2008 was carried out. We compared patients with or without major bleeding in relation to pre-procedure clinic and angiographic characteristics and in-hospital and late outcomes. Results: Major bleeding was identified in 1.6\% of the patients. A multiple logistic regression model identified as independent predictors of major bleeding: female sex, age, previous coronary bypass surgery and use of glycoprotein Ilb/IIla inhibitors. Major bleeding was an independent predictor of in-hospital acute renal failure, myocardial infarction and death and of myocardial infarction in the late follow-up. Conclusion: Our study demonstrates that procedure-related major bleeding is an independent predictor of acute and late adverse events post-PCI.

DESCRIPTORS: Hemorrhage. Angioplasty, transluminal, percutaneous coronary. Mortality.

\footnotetext{
1 Hospital Beneficência Portuguesa - São Paulo, SP.

Correspondência: José Armando Mangione. Rua Maestro Cardim, 769 - 10 SS - sala 71 - Bloco 1 - São Paulo, SP - CEP 01323-900 E-mail: uci@uol.com.br

Recebido em: 30/7/2008 • Aceito em: 12/11/2008
}

O sangramento maior (SM) periprocedimento é uma das mais freqüentes complicações associadas à intervenção coronária percutânea (ICP), com incidência variando de $2,2 \%$ a $14 \%{ }^{1}$. Dados de estudos retrospectivos e prospectivos indicam que a hemorragia está associada ao aumento de mortalida$\mathrm{de}^{2,3}$, evidenciando a importância de minimizar esse tipo de intercorrência e não apenas as complicações isquêmicas que possam ocorrer durante e após o tratamento percutâneo. Chamado de desfecho quádruplo, porque inclui os três eventos tradicionais de avaliação 
de segurança da ICP (morte, infarto do miocárdio [IAM] e necessidade de revascularização urgente em trinta dias) mais sangramento, essa definição foi usada no estudo REPLACE-2 para avaliar os resultados dos pacientes submetidos a revascularização percutânea e demonstrou que o SM é preditor independente de mortalidade em um ano após ICP tanto eletiva como urgente ${ }^{4}$.

A hipoperfusão conseqüente às complicações hemorrágicas afeta as funções renal e hepática, causando efeitos adversos no sistema de coagulação e nas plaquetas. Ocorre também aumento da necessidade de transfusão sanguínea, que é preditor independente de mortalidade hospitalar e em um ano, com forte associação entre número de unidades trans-

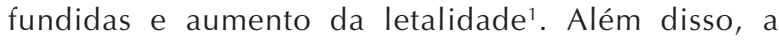
hemorragia periprocedimento está associada a aumento do risco de complicações isquêmicas recorrentes, afetando a sobrevivência a curto e longo $\operatorname{prazos}^{5}$.

Em decorrência desses fatos, o objetivo deste estudo foi avaliar os preditores de sangramento maior e o impacto deste na ocorrência de eventos adversos em pacientes submetidos a ICP em nosso Serviço.

\section{MÉTODO}

Realizada análise retrospectiva de nosso banco de dados no período compreendido entre junho de 1997 e fevereiro de 2008, identificando-se 8.739 pacientes submetidos a ICP eletiva. Foram incluídos os portadores de doença arterial coronária assintomáticos com prova isquêmica positiva, angina estável e também aqueles com síndrome coronária aguda sem elevação do segmento ST, podendo ser tratadas lesões de novo e também as reestenoses, assim como lesões em enxertos arteriais e venosos, além daqueles com disfunção ventricular esquerda. Foram excluídos apenas os pacientes com síndrome coronária aguda com elevação do segmento ST (angioplastia primária). Comparamos os pacientes que apresentaram SM aos que não apresentaram (NSM) em relação às características clínicas, angiográficas e do procedimento, e sua evolução hospitalar e tardia.

A técnica de revascularização empregada foi angioplastia com balão do início do estudo até dezembro de 1999, quando os stents não-farmacológicos foram incorporados à prática da cardiologia intervencionista no sistema público de Saúde. A partir dessa data, a ICP com o balão ficou restrita a casos específicos, como a abordagem de vasos muito finos $(<2,0 \mathrm{~mm})$, lesões reestenóticas e tratamento de bifurcações nos casos que não necessitavam de stent no ramo lateral. Posteriormente, a introdução dos stents farmacológicos aprovados pelo Food and Drug Administration (FDA) e pela Agência Nacional de Vigilância Sanitária (ANVISA) permitiu a utilização dessas próteses em nosso meio a partir de abril de 2003.

As vias de acesso utilizadas foram: a braquial, particularmente no início do estudo (3,5\% grupo SM vs. 2,2\% grupo NSM), em que a hemostasia era realizada com a sutura da artéria dissecada; a via femoral (95,1\% grupo SM vs. 93,7\% grupo NSM), com a hemostasia obtida por meio de compressão manual da artéria; e também a via radial (1,4\% grupo SM vs. 3,5\% grupo NSM), na qual a hemostasia era realizada com a retirada do introdutor na sala de hemodinâmica, com aplicação de curativo compressivo de forma a envolver toda a circunferência do punho.

Todos os pacientes receberam heparina nãofracionada no momento da ICP, na dose de $70 \mathrm{UI} / \mathrm{kg}$ a $100 \mathrm{UI} / \mathrm{kg}$. Administrou-se ácido acetilsalicílico na dose de $200 \mathrm{mg} /$ dia para os pacientes que já o utilizavam cronicamente e $500 \mathrm{mg}$, pelo menos 24 horas antes da ICP, naqueles que não faziam uso desse fármaco. Em todos os casos, a droga foi mantida indefinidamente após a ICP. O clopidogrel foi utilizado na dose de $75 \mathrm{mg} /$ dia pelo menos sete dias antes do procedimento ou com dose de ataque de $300 \mathrm{mg}$ nos que não fizeram uso prévio ao procedimento. Posteriormente, os pacientes eram orientados a tomar dose de 75 mg/dia de acordo com o tipo do stent implantado: não-farmacológico (trinta dias) ou farmacológico (três meses a doze meses).

O uso do inibidor da glicoproteína Ilb/Illa ficou a critério do cardiologista intervencionista, levando-se em consideração as características clínicas e angiográficas do paciente ou o surgimento de complicações periprocedimento. O fármaco utilizado em nosso Serviço foi o abciximab, na dose de 0,25 mg/kg em bolus, seguido por $0,125 \mathrm{mcg} / \mathrm{kg} / \mathrm{min}$ (máximo de $10 \mathrm{mcg} / \mathrm{min}$ ) infundido por doze horas.

No acompanhamento clínico, os pacientes foram avaliados por consulta médica ou por contato telefônico aos trinta dias, seis meses e um ano após ICP. A partir de então eram avaliados anualmente.

\section{Definições dos eventos adversos}

O SM após ICP foi definido pelos critérios do TIMI (Thrombolysis In Myocardial Infarction) ${ }^{6}$ : presença de sangramento intracraniano ou sinais clínicos de hemorragia associada a queda dos níveis de hemoglobina de mais de 5,0 g/dl (ou queda absoluta do hematócrito de pelo menos $15 \%$ ).

Eventos cardíacos adversos maiores (ECAM) intrahospitalares foram definidos como morte, IAM e necessidade de nova revascularização urgente do vasoalvo. ECAM em um ano foram definidos como morte, IAM e necessidade de nova revascularização do vasoalvo, cirúrgica ou percutânea. 
IAM foi definido como o desenvolvimento de nova onda Q em duas ou mais derivações eletrocardiográficas contíguas ou elevação da CK-MB maior que três vezes o limite superior da normalidade.

O sucesso angiográfico foi definido como lesão residual menor que $50 \%$ e fluxo TIMI 3 nos pacientes tratados com angioplastia com balão e lesão residual menor que $20 \%$ e fluxo TIMI grau 3 nos tratados com stent.

O sucesso do procedimento foi definido como o sucesso angiográfico na ausência de complicações maiores (óbito, IAM ou necessidade de nova revascularização urgente) ${ }^{7}$.

\section{Análise estatística}

A análise dos dados foi feita com o pacote estatístico R versão 2.6.0 ( $R$ Development Core Team, 2007). Todos os testes foram realizados considerando hipóteses bilaterais e assumindo nível de significância alfa $=5 \%$.

Inicialmente foi utilizada a estatística descritiva para avaliar freqüência, média e desvio padrão das variáveis de interesse.

Foi realizada uma análise univariada para a comparação dos grupos SM e NSM. Para as variáveis contínuas, as comparações das médias foram realizadas através do teste-t para amostras independentes. Todos os requisitos necessários para a aplicação do teste-t foram satisfeitos, garantindo assim a confiabilidade dos resultados obtidos. Os testes de qui-quadrado ou exato de Fisher foram utilizados para comparar as variáveis qualitativas. O teste exato de Fisher foi aplicado quando as suposições para o teste de qui-quadrado não foram atendidas.
Em seguida à análise univariada, foi conduzida regressão logística múltipla (análise multivariada) para verificar os efeitos das variáveis em conjunto, objetivando determinar os preditores de sangramento e a influência deste sobre a evolução dos pacientes.

Os resultados da regressão logística encontram-se expressos em odds ratio (OR) e intervalo de confiança de $95 \%$ (IC 95\%).

\section{RESULTADOS}

Neste estudo foram analisados 8.739 pacientes consecutivos com 11.875 lesões tratadas e 9.148 stents implantados (média de 1,04 stent/paciente), tendo $138(1,6 \%)$ desses pacientes apresentado SM.

Em relação às características clínicas e demográficas (Tabela 1), observamos que, nos pacientes que apresentaram SM, houve predominância do sexo feminino $(59,4 \%$ vs. $32,9 \%$; p < 0,001), dos pacientes mais idosos $(67,91 \pm 10,71$ anos vs. 61,26 \pm 11,26 anos; $\mathrm{p}<0,001$ ) e cirurgia de revascularização (RM) prévia $(18,8 \%$ vs. $12,5 \%$; p $=0,025)$. Por outro lado, não foram observadas diferenças em relação ao índice de massa corporal (IMC) nos dois grupos (SM $26 \pm$ 4,61 vs. NSM $26 \pm 4,45 ; p=0,892$ ) ou ao quadro clínico de apresentação.

Em relação às características angiográficas e do procedimento (Tabela 2), não ocorreram diferenças entre os grupos no que se refere às artérias tratadas, ao tipo de lesão, à extensão da doença coronária, à presença de disfunção ventricular esquerda e à taxa de sucesso angiográfico. Entretanto, constatamos diferenças no uso de inibidores da glicoproteína IIb/IIla (IGP Ilb /IIla), os quais foram utilizados com maior freqüência no grupo SM $(10,9 \%$ vs. $4,8 \%$; $p=0,001)$.

TABELA 1

Características clínicas e demográficas

\begin{tabular}{lccc}
\hline & $\begin{array}{c}\text { Sangramento maior } \\
(\mathbf{n}=\mathbf{1 3 8})\end{array}$ & $\begin{array}{c}\text { Sem sangramento maior } \\
(\mathbf{n}=\mathbf{8 . 6 0 1})\end{array}$ & $\mathbf{p}$ \\
\hline Média de idade, anos & $67,91 \pm 10,71$ & $61,26 \pm 11,26$ & $<, 001$ \\
Sexo feminino & $82(59,4 \%)$ & $2834(32,9 \%)$ & 0,001 \\
Hipertensão arterial & $107(77,5 \%)$ & $6.618(76,9 \%)$ & 0,866 \\
Diabetes melito & $33(23,9 \%)$ & $1.998(23,2 \%)$ & 0,849 \\
Tabagismo & $26(18,8 \%)$ & $2237(26 \%)$ & 0,057 \\
RM prévia & $26(18,8 \%)$ & $1.072(12,5 \%)$ & 0,025 \\
Quadro clínico & $15(10,9 \%)$ & $1.439(16,7 \%)$ & 0,054 \\
$\quad$ Assintomático & $73(52,9 \%)$ & $4722(54,9 \%)$ & $2442(28,4 \%)$ \\
$\quad$ Angina estável & $50(36,2 \%)$ & $26 \pm 4,45$ & 0,892 \\
Angina instável & $26 \pm 4,61$ & &
\end{tabular}

IMC = Índice de massa corporal; $\mathrm{n}$ = número de pacientes; $\mathrm{RM}$ = revascularização do miocárdio. 
Dall'Orto CC, et al. Incidência, Preditores e Impacto Clínico dos Sangramentos Maiores Associados à Intervenção Coronária Percutânea. Rev Bras Cardiol Invas. 2008;16(4):439-444.

TABELA 2

Características angiográficas e do procedimento

\begin{tabular}{|c|c|c|c|}
\hline & $\begin{array}{c}\text { Sangramento maior }(n=138) \\
194 \text { procedimentos }\end{array}$ & $\begin{array}{c}\text { Sem sangramento maior }(n=8.601) \\
11.681 \text { procedimentos }\end{array}$ & $\mathbf{p}$ \\
\hline Vaso tratado & & & 0,133 \\
\hline Artéria coronária direita & $53(27,3 \%)$ & $3.614(30,9 \%)$ & \\
\hline Artéria descendente anterior & $74(38,5 \%)$ & $4.100(35,1 \%)$ & \\
\hline Diagonal & $10(5,1 \%)$ & $469(4 \%)$ & \\
\hline Artéria circunflexa & $21(10,8 \%)$ & $1.441(12,3 \%)$ & \\
\hline Obtuso marginal & $17(8,7 \%)$ & $1.361(11,6 \%)$ & \\
\hline Enxerto de veia safena & $13(9,1 \%)$ & $461(5,3 \%)$ & \\
\hline Enxertos arteriais & $1(0,5 \%)$ & $75(0,8 \%)$ & \\
\hline Tipo de lesão & & & 0,729 \\
\hline$A+B 1$ & $70(36 \%)$ & $4.351(37,2 \%)$ & \\
\hline B2 & $72(37,2 \%)$ & $4.020(34,4 \%)$ & \\
\hline C & $52(26,8 \%)$ & $3.310(28,4 \%)$ & \\
\hline Número de vasos acometidos & & & 0,892 \\
\hline Uniarteriais & $61(44,2 \%)$ & $3.956(46,2 \%)$ & \\
\hline Biarteriais & $58(42 \%)$ & $3.447(40,2 \%)$ & \\
\hline Triarteriais & $19(13,8 \%)$ & $1.161(13,6 \%)$ & \\
\hline Disfunção grave do VE* & $6,7(4,9)$ & $275(3,2 \%)$ & 0,234 \\
\hline Uso de IGP IIb/IIIa* & $15(10,9 \%)$ & $416(4,8 \%)$ & 0,001 \\
\hline Sucesso angiográfico* & $134(97,1 \%)$ & $8.592(97,7 \%)$ & 0,231 \\
\hline
\end{tabular}

Na evolução hospitalar (Tabela 3), obtivemos taxas de sucesso angiográfico semelhantes nos dois grupos $(97,1 \%$ no grupo SM e $97,7 \%$ no grupo NSM; $p=0,775)$. Entretanto, no grupo SM verificamos taxas significativamente maiores de insuficiência renal aguda $(6,5 \%$ vs. $1,1 \% ; p<0,001)$ e também de eventos cardíacos maiores $(5,8 \%$ vs. $2,2 \% ; p=0,012)$, à custa de óbito $(2,2 \%$ vs. $0,4 \%$; p $=0,022)$ e IAM $(4,3 \%$ vs. $1,5 \% ; p=0,017)$, comparativamente ao grupo NSM.

A Tabela 4 mostra os preditores de ocorrência de SM pós-ICP. De acordo com o modelo de regressão logística múltipla, o risco de SM foi mais elevado para o sexo feminino (OR = 2, IC 95\% 1,97-4,02), cirurgia de $R M$ prévia $(O R=1,88$, IC 95\% 1,16-3,02) e uso de IGP IIb/IIIa (OR = 2,19, IC 95\% 1,25-3,83). Adicionalmente, em relação à idade, observamos que, para o acréscimo de um ano de vida do paciente, a chance de sangramento aumentou em 5\%.

Na Tabela 5, a análise do modelo de regressão logística revela que a presença de SM pós-ICP eleva os riscos do desenvolvimento de insuficiência renal aguda (IRA) e também de ECAM, à custa de IAM e óbito, na fase hospitalar.

No seguimento tardio (Tabela 6), 90,7\% dos pacientes foram acompanhados num período médio de
500 dias $\pm 621,24$ dias. Encontramos maiores taxas de ECAM $(29,8 \%$ vs. $21,3 \% ; p=0,022)$, à custa de IAM $(6,5 \%$ vs. $2,9 \%$; p $=0,018)$, para o grupo SM. No entanto, não foram observadas diferenças em relação à necessidade de nova revascularização do vaso-alvo $(15,3 \%$ vs. $13,2 \% ; p=0,495)$ ou óbito $(8,9 \%$ vs. $5,8 \% ; p=0,142)$ entre os dois grupos.

Quando aplicamos o modelo de regressão logística, o risco de IAM nos pacientes com sangramento foi maior (OR $=2,43$, IC 95\% 1,17-5,04), assim como o risco de ECAM $(\mathrm{OR}=1,57, \mathrm{IC} 95 \%$ 1,06-2,31) (Tabela 7).

\section{DISCUSSÃO}

Os principais achados desta pesquisa envolvendo 8.739 pacientes consecutivos e não-selecionados submetidos a ICP foram:

- fatores preditores independentes para a ocorrência de SM pós-ICP foram sexo feminino, idade, cirurgia de RM prévia e uso de IGP IIb/IIla;

- SM periprocedimento foram preditores independentes de IRA e ECAM intra-hospitalar e de ECAM no seguimento tardio.

Recentemente, vários estudos têm destacado o problema do sangramento pós-ICP, e muitos pesquisa- 
Dall'Orto CC, et al. Incidência, Preditores e Impacto Clínico dos Sangramentos Maiores Associados à Intervenção Coronária Percutânea. Rev Bras Cardiol Invas. 2008;16(4):439-444.

TABELA 3

Evolução hospitalar

\begin{tabular}{lccr}
\hline & $\begin{array}{c}\text { Sangramento maior } \\
(\mathbf{n}=\mathbf{1 3 8})\end{array}$ & $\begin{array}{c}\text { Sem sangramento maior } \\
(\mathbf{n = ~ 8 . 6 0 1 )}\end{array}$ & $\mathbf{p}$ \\
\hline Sucesso angiográfico & $134(97,1 \%)$ & $8.405(97,7 \%)$ & 0,775 \\
ECAM & $8(5,8 \%)$ & $187(2,2 \%)$ & 0,012 \\
Óbito & $3(2,2 \%)$ & $34(0,4 \%)$ & 0,022 \\
IAM & $6(4,3 \%)$ & $125(1,5 \%)$ & 0,017 \\
RVA urgente & $0(0 \%)$ & $31(0,36 \%)$ & 1,000 \\
IRA & $9(6,5 \%)$ & $91(1,1 \%)$ & $<0,001$ \\
\hline
\end{tabular}

ECAM = eventos cardíacos adversos maiores; IAM = infarto agudo do miocárdio; IRA = insuficiência renal aguda; $\mathrm{n}=$ número de pacientes; RVA = revascularização do vaso-alvo.

TABELA 4

Preditores de ocorrência de sangramentos maiores

\begin{tabular}{lcc}
\hline & \multicolumn{1}{c}{$\mathbf{p}$} & $\begin{array}{c}\mathbf{O R} \\
\text { (IC 95\%) }\end{array}$ \\
\hline Idade & $<0,001$ & 1,05 \\
Sexo feminino & $<0,001$ & $(1,03-1,07)$ \\
& 2,80 \\
Cirurgia de RM prévia & 0,009 & $(1,97-4,02)$ \\
& & 1,88 \\
Uso de IGP IIIb/IIla & 0,006 & $(1,16-3,02)$ \\
& & 2,19 \\
& & $(1,25-3,83)$
\end{tabular}

IC $95 \%$ = intervalo de confiança de 95\%; IGP = inibidores da glicoproteína; $\mathrm{OR}=$ odds ratio (razão de chances); $\mathrm{RM}=$ revascularização do miocárdio.

dores defendem a inclusão das complicações hemorrágicas periprocedimento como uma variável importante a ser considerada como evento adverso, tanto aos trinta dias como em um ano, constituindo dessa forma o chamado desfecho quádruplo após o tratamento percutâneo (morte, IAM, revascularização de urgência do vaso-alvo e sangramento maior aos trinta dias) $)^{4,8,9}$.

Historicamente, a redução dos eventos isquêmicos, alcançada por meio da utilização de poderosos agentes antitrombóticos e antiplaquetários, tem sido acompanhada por aumento dos fenômenos hemorrágicos ${ }^{8}$. Nesses casos, a ocorrência de hemorragia pode levar à necessidade de interrupção das terapias acima mencionadas, expondo o paciente a risco elevado de complicações isquêmicas por trombose do stent. Paralelamente, algumas pesquisas revelam que a necessidade de transfusão sanguínea está associada a aumento da mortalidade intra-hospitalar e também ao final de um ano, independentemente da categoria de sangramento ${ }^{8,10}$, além do óbvio impacto econômico.
TABELA 5

Presença de sangramentos maiores e ocorrência de eventos adversos na fase hospitalar

\begin{tabular}{lcc}
\hline Sangramento maior & $\mathbf{p}$ & $\begin{array}{c}\text { OR } \\
(\text { IC 95\%) }\end{array}$ \\
\hline ECAM & 0,001 & 3,17 \\
Óbito & 0,042 & $(1,59-6,34)$ \\
& & 3,77 \\
IAM & 0,018 & $(1,04-13,6)$ \\
RVA & & 2,79 \\
(cirúrgica/percutânea) & 0,998 & $(1,19-6,54)$ \\
IRA & $<0,001$ & 0,01 \\
& & $(2,79-11,87)$ \\
& & 5,75
\end{tabular}

ECAM = eventos cardíacos adversos maiores; IC 95\% = intervalo de confiança de 95\%; IRA = insuficiência renal aguda; $\mathrm{OR}=$ odds ratio (razão de chances); $\mathrm{RVA}=$ revascularização do vaso-alvo.

Os resultados encontrados em nosso estudo estão em concordância com aqueles reportados pelo REPLACE- ${ }^{4}$, que apontou idade avançada, sexo feminino e utilização de IGP IIb/IIla como preditores independentes para a ocorrência de SM. Essas características, facilmente identificáveis, são úteis para detectarmos pacientes de risco, antes da administração da terapêutica antitrombótica.

Nossos achados também estão em concordância com outros, como os de Feit et al. ${ }^{8}$, que evidenciaram maior taxa de mortalidade aos trinta dias e em um ano em pacientes com SM e Manoukian et al. ${ }^{11}$, com maior índice de eventos isquêmicos e óbito aos trinta dias nos pacientes com complicações hemorrágicas.

Apesar de as taxas de sangramento periprocedimento terem diminuído muito nos últimos dez anos ${ }^{1}$, medidas para sua redução adicional permanecem como 
Dall'Orto CC, et al. Incidência, Preditores e Impacto Clínico dos Sangramentos Maiores Associados à Intervenção Coronária Percutânea. Rev Bras Cardiol Invas. 2008;16(4):439-444.

TABELA 6

Eventos clínicos no seguimento tardio

\begin{tabular}{|c|c|c|c|}
\hline & $\begin{array}{l}\text { Sangramento maior } \\
\qquad(\mathrm{n}=138)\end{array}$ & $\begin{array}{l}\text { Sem sangramento maior } \\
(\mathbf{n}=\mathbf{8 . 6 0 1})\end{array}$ & $\mathbf{p}$ \\
\hline Pacientes seguidos & $124(96,8 \%)$ & $7433(90,6 \%)$ & 0,072 \\
\hline Tempo de seguimento & $481 \pm 608,72$ & $519 \pm 633,76$ & 0,980 \\
\hline ECAM & $37(29,8 \%)$ & $1585(21,3 \%)$ & 0,022 \\
\hline Óbito & $11(8,9 \%)$ & $428(5,8 \%)$ & 0,142 \\
\hline IAM & $8(6,5 \%)$ & $212(2,9 \%)$ & 0,018 \\
\hline RVA & $19(15,3 \%)$ & $983(13,2 \%)$ & 0,495 \\
\hline
\end{tabular}

ECAM = eventos cardíacos adversos maiores; IAM = infarto agudo do miocárdio; $\mathrm{n}$ = número de pacientes; $\mathrm{RVA}=$ revascularização do vaso-alvo.

TABELA 7

Presença de sangramentos maiores e ocorrência de eventos clínicos na evolução tardia

\begin{tabular}{lcc}
\hline & $\mathbf{p}$ & $\begin{array}{c}\text { OR } \\
(\mathbf{I C} \mathbf{9 5} \%)\end{array}$ \\
\hline ECAM & 0,023 & $\begin{array}{c}1,57 \\
(1,06-2,31) \\
1,71\end{array}$ \\
Óbito & 0,096 & $(0,91-3,23)$ \\
& & 2,43 \\
IAM & 0,018 & $(1,17-5,04)$ \\
& 0,998 & 0,42 \\
RVA & & $(0,75-2,01)$ \\
& & \\
\hline $\begin{array}{l}\text { ECAM = eventos cardíacos adversos maiores; IC 95\% } \\
\text { intervalo de confiança de 95\%; OR = odds ratio (razão de } \\
\text { chances); RVA = revascularização do vaso-alvo. }\end{array}$ \\
\hline
\end{tabular}

um importante objetivo terapêutico, tornando indispensáveis melhorias no tratamento clínico combinadas com a observação cuidadosa dos pacientes, principalmente aqueles de alto risco.

As limitações deste estudo incluem o fato de esta ter sido uma análise retrospectiva de um banco de dados envolvendo diferentes períodos de tratamento na prática da cardiologia intervencionista, em particular dos diferentes regimes antiagregantes e anticoagulantes adjuntos.

\section{CONCLUSÃO}

Este estudo demonstra que o SM periprocedimento é preditor independente de IAM, óbito e ECAM na evolução intra-hospitalar e de IAM e ECAM no seguimento clínico tardio. O reconhecimento de que as complicações hemorrágicas têm importância similar à das complicações isquêmicas em relação aos eventos adversos é fundamental para o planejamento e a otimização da ICP, particularmente nos pacientes de risco.

\section{REFERÊNCIAS BIBLIOGRÁFICAS}

1. Kinnaird TD, Stabile E, Mintz GS, Lee CW, Canos DA, Gevorkian $\mathrm{N}$, et al. Incidence, predictors, and prognostic implications of bleeding and blood transfusion following percutaneous coronary interventions. Am J Cardiol. 2003;92(8):930-5.

2. ESPRIT Investigators. Enhanced Suppression of the Platelet IIb/IIla Receptor with Integrilin Therapy. Novel dosing regimen of eptifibatide in planned coronary stent implantation (ESPRIT): a randomized, placebo-controlled trial. Lancet. 2000;356(9247):2037-44.

3. Moscucci M, Fox KA, Cannon CP, Klein W, López-Sendón J, Montalescot G, et al. Predictors of major bleeding in acute coronary syndromes: the Global Registry of Acute Coronary Events (GRACE). Eur Heart J. 2003;24(20):1815-23.

4. Lincoff AM, Bittl JA, Harrington RA, Feit F, Kleiman NS, Jackman $\mathrm{JD}$, et al. Bivalirudin and provisional glycoprotein Ilb/llla blockade compared with heparin and planned glycoprotein IIb/IIla blockade during percutaneous coronary intervention: REPLACE2 randomized trial. JAMA. 2003;289(7):853-63.

5. Segev A, Strauss BH, Tan M, Constance C, Langer A, Goodman SG; Canadian Acute Coronary Syndromes Registries Investigators. Predictors and 1-year outcome of major bleeding in patients with non-ST-elevation acute coronary syndromes: insights from the Canadian Acute Coronary Syndrome Registries. Am Heart J. 2005;150(4):690-4.

6. TIMI Study Group. Definitions used in TIMI trials. Disponível em: http://www.timi.org. Acesso em: 20/2/2008.

7. ACC/AHA/SCAI 2005 Guideline Update for Percutaneous Coronary Intervention. Disponível em: http://www.acc.org.

8. Feit F, Voeltz MD, Attubato MJ, Lincoff AM, Chew DP, Bittl $\mathrm{JA}$, et al. Predictors and impact of major hemorrhage on mortality following percutaneous coronary intervention from the REPLACE-2 Trial. Am J Cardiol. 2007;100(9):1364-9.

9. Ndrepepa G, Berger PB, Mehilli J, Seyfarth M, Neumann FJ, Schömig A, et al. Periprocedural bleeding and 1-year outcome after percutaneous coronary interventions: appropriateness of including bleeding as a component of a quadruple end point. J Am Coll Cardiol. 2008;51(7):690-7.

10. Rao SV, Jollis JG, Harrington RA, Granger CB, Newby LK, Armstrong PW, et al. Relationship of blood transfusion and clinical outcomes in patients with acute coronary syndromes. JAMA. 2004;292(13):1555-62.

11. Manoukian SV, Feit F, Mehran R, Voeltz MD, Ebrahimi R, Hamon $M$, et al. Impact of major bleeding on 30-day mortality and clinical outcomes in patients with acute coronary syndromes: an analysis from the ACUITY Trial. J Am Coll Cardiol. 2007;49(12):1362-8. 